\title{
Multi-DOF Forceps Manipulator System for Laparoscopic Surgery - Mechanism Miniaturized \& Evaluation of New Interface -
}

\author{
Ryoichi Nakamura', Takeshi Oura ${ }^{2}$, Etsuko Kobayashi², Ichiro Sakuma ${ }^{2}$, \\ Takeyoshi Dohi ${ }^{2}$, Naoki Yahagi ${ }^{2}$, Takayuki Tsuji ${ }^{2}$, Mitsuo Shimada ${ }^{3}$, \\ Makoto Hashizume ${ }^{4}$ \\ ${ }^{1}$ Dept. of Precision Engineering, Graduate School of Engineering, \\ ${ }^{2}$ Institute of Environmental Studies, Graduate school of Frontier Science, \\ the Univ. of Tokyo, 7-3-1, Hongo, Bunkyo-ku, Tokyo 113-8656 Japan \\ \{ryoichi, takeshi, etsuko, sakuma, dohi, yahagi, \\ tsuji\}@miki.pe.u-tokyo.ac.jp \\ ${ }^{3}$ Department of Surgery and Science, Graduate School of Medical Sciences, \\ ${ }^{4}$ Dept. of Disaster and Emergency Medicine, Grad. School of Medical Science, \\ Kyushu University, 3-1-1, Maidashi, Higashi-ku, Hukuoka 812-8582 Japan \\ mshimadaesurg2.med.kyushu-u.ac.jp \\ mhashi@dem.med.kyushu-u.ac.jp
}

\begin{abstract}
The Multi-DOF forceps manipulator we developed has two additional DOF of bending on the tip of forceps, and provides new surgical fields and techniques for surgeons. The most remarkable characteristics of the prototype described in this paper are: 1) the small diameter and the small radius of curvature of bending; 2) the confirmation of perfect cleanness and sterilization of this manipulator. In this paper, we will show some new mechanisms of the forceps manipulator. Firstly, we made new mechanism of bending forceps. Using the mechanism, we made new prototype of forceps manipulator which diameter is $5 \mathrm{~mm}$. Secondly, we developed new concept of man-machine interface for the system. It will show the new control method and surgeons can operate surgeries with more dexterity and without confusion. We evaluated the system including new interface on typical laparosurgical procedure using simulator, and confirmed the effectiveness of this concepts.
\end{abstract}

\section{Introduction}

Laparoscopic surgery is now widely established as minimal invasive surgery (MIS). However, it has some problems and difficulties due to the "minimal invasive" method. The forceps and laparoscope are inserted into the visceral cavity through the trochars that are fixed on the abdominal wall. These tools have only four degrees of freedom of movement (DOF) and small working areas (Fig. 1).

Using conventional tools (forceps), surgeons can approach an operation point only through a single approach path. However, in some cases, various alternative approach paths for an operation area are needed. The tools thus require additional DOF to solve these problems (Fig. 2). 


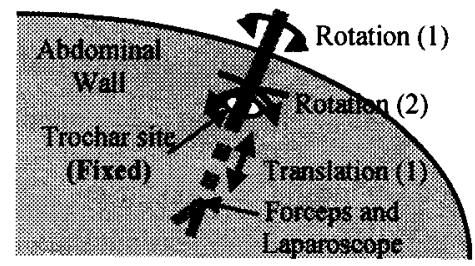

Fig. 1. Four DOF of surgical tools in laparoscopic surgery

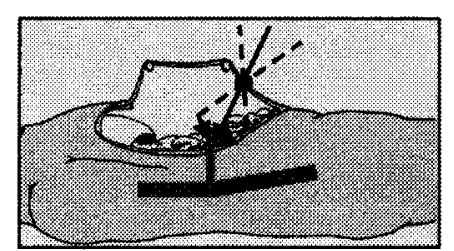

Fig. 2. Approach path of surgical tools

$\longleftarrow$ is approach path of conventional tools. $4--$ are approach paths of tools with additional DOF.

Surgical robots, which can help surgeons' manipulation such as steadier holding, more precise operating, and remote control, are being studied and developed. However, there are many problems in the use of robots in medical fields. The most important consideration must be safety, both in terms of engineering and medical effectiveness. Recently, forceps with additional DOF for MIS have been studied in many institutes and applied to many actual cases [1][2][3][4][5][8], but there is a problem with the following points;

- Diameters of Forceps with additional DOF are still big

The major diameter of commercial forceps for laparoscopic surgery is $5 \mathrm{~mm}$ and is gradually smaller, but the major diameter of the forceps with additional DOF that has been studied in many institutes is around $10 \mathrm{~mm}$.

- Sizes of the whole system of Forceps with additional DOF are still big

Big space around the operation table in operation room is needed for the system and it cause the problems about the coexistence with doctor and safety.

Taking these problems into account, we developed Multi-DOF Forceps Manipulator System for laparoscopic surgery [6].

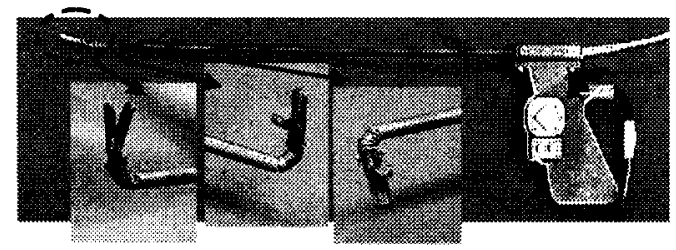

Fig. 3. First Prototype of Multi-DOF forceps manipulator

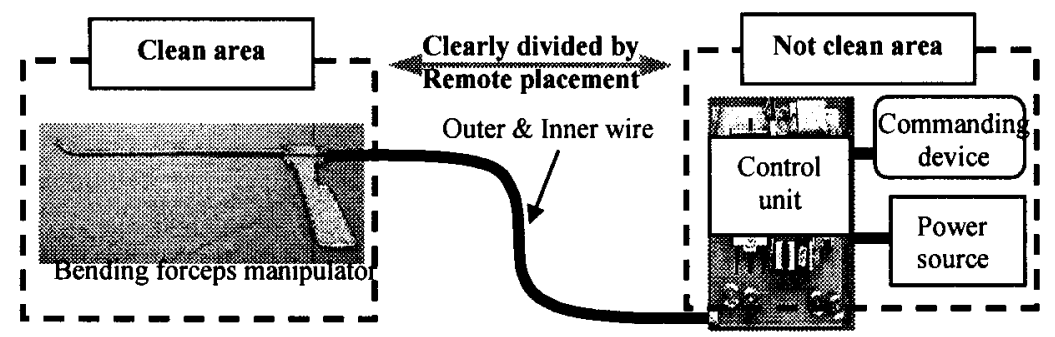

Fig. 4. Total system components of the Multi-DOF forceps manipulator.

This Forceps manipulator has $6 \mathrm{~mm}$ diameter and the characteristic of less-spaceconsuming that is achieved by the divided architecture of forceps \& actuators. In this 
paper, we present new two mechanisms of Multi-DOF forceps manipulator system. The first mechanism is the bending mechanism of $\phi 5 \mathrm{~mm}$. The second one is the new man-machine interface of the manipulator system. These mechanisms are important features to realize the more evolutional surgery. The methods used in order to construct the mechanisms are presented in Section 2. The experimental results are presented in Section 3, including the measurement of mechanical characteristics and the performance of this manipulator in surgical environments. Using this manipulator, problems in laparoscopic surgery are clearly solved, and new standards with higher effectiveness and safety are achieved.

\section{Method}

\subsection{Bending Mechanism}

We developed the Multi-DOF forceps manipulator, which provides two additional DOF of bending. The bending mechanism is composed of four stainless steel rings with a coupling giving one DOF, stainless wire, and Teflon tubes (shown in Fig. 5 and 6). This mechanism is driven by four stainless steel wires. The ranges of bending motion are 0 to 90 degrees for each two DOF. [6]

The important problem on miniaturizing

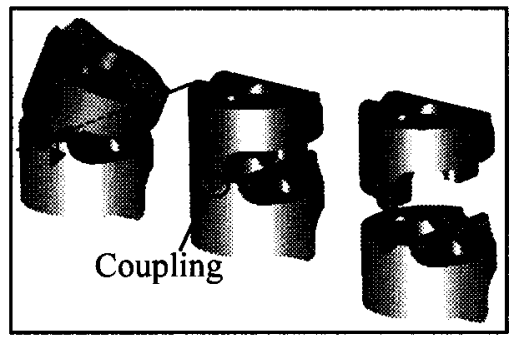

Fig. 5. Stainless steel ring joint mechanism is to have to maintain strength / hardness of joint and diameter of wire though we decrease total size. In this study we miniaturized the mechanism by detaching the wire conservation with a Teflon tube.

In addition, we got drive range in former $\phi 6 \mathrm{~mm}$ diameter Forceps Manipulator System by establishing two joints for each DOF (drive range of 1 joint: $45 \mathrm{deg} * 2=$ $90 \mathrm{deg}$ ). However, I considered a fall of efficiency / outbreak power of the wire with mechanism miniaturized and produced the joint that had big drive range of $60 \mathrm{deg}$.

\subsection{New Man-Machine Interface}

Surgeons use forceps in an operation with one hand. Therefore, they can control all movement with one hand even if the degree of freedom and input devices that control the DOF increase by bending mechanism that we developed in this study.

In the last year system we developed, we possess the input device which a joy-stick button for bending DOF and two buttons for grasping DOF were built in to a grip as man machine interface to operate the 3 degree of freedom on the tip of Multi-DOF Forceps Manipulator System. (Fig. 6) However, in this device, some problems were figured through tests and evaluations.

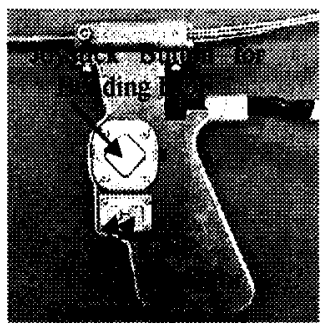

Fig. 6. Button Interface for Multi-DOF Forceps 
(1) It is difficult to operate bending and grasping move quickly and without confusion because there are many buttons in small area.

(2) It is hard to do operation of intuitionally because the arrangement of direction of buttons and a direction of bending axes on the tip of forceps do not correspond.

(3) There are no correspondence between input value and output value. (Input value is time to push the button / Output value is degree of bending)

(4) It is difficult to use more than one DOF simultaneously and coordinatively (synchronized drive).

Development of better interface is much necessary in order to do complicated procedures in laparosurgery (e.g. suturing, ligation, etc.) using this Multi-DOF Forceps Manipulator. In addition, in this manipulator, it is different from major surgical robot systems (Master-slave manipulator) in the point that surgeons maintain forceps manipulator by their own hand and operate the DOF on the tip part as well. It offers the following advantage to surgeons;

1. Surgeon keeps his haptic sense during the surgery even if he uses manipulator. (In many cases, the lack of haptic sense is main consideration in developing surgical manipulator)

2. The time / spacial / economical cost of this manipulator is much more few than the cost of the surgical robot system which control the whole DOF for controlling forceps (7DOF or more)

Therefore, simple and small interface to reflect intention of surgeons at sight becomes necessary to establish the Multi-DOF Forceps Manipulator System which keeps these advantages.

In this study, we developed the new man-machine interface mechanism. On this interface, surgeons drive the two bending axes using the two bending axes of their first finger. (shown in Fig. 7)
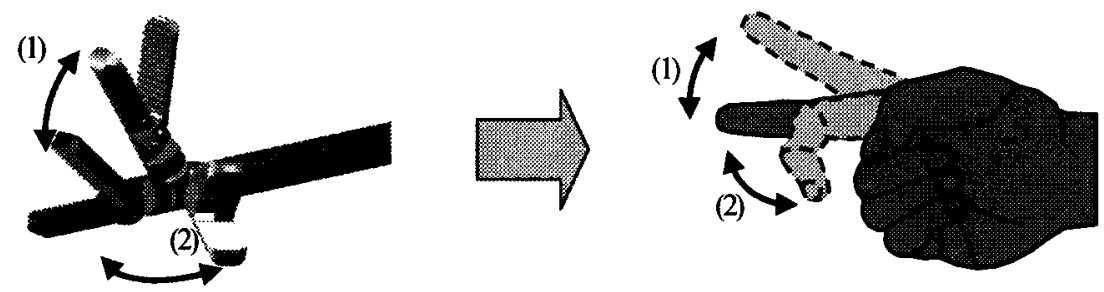

Fig. 7. DOF Comparison between the bending forceps and the first finger

This system is the technique that we paid its attention to bending movement of a first finger. Bending movement of a first finger corresponds to bending axis (2) of Forceps Manipulator, and up-and-down motion product of a first finger corresponds to bending axis (1) of Forceps Manipulator. We think that there is a little influence that operation with a first finger gives to a stability maintenance state of a grip, because the part, which is actually concerned with holding the grip, is three fingers (middle finger / third finger / little finger) and the part of the root of thumb. A first finger is not concerned greatly when I grasp a thing, hence the interface using it is appropriate for this manipulator.

The prototype of the new interface is shown in Fig.8. 


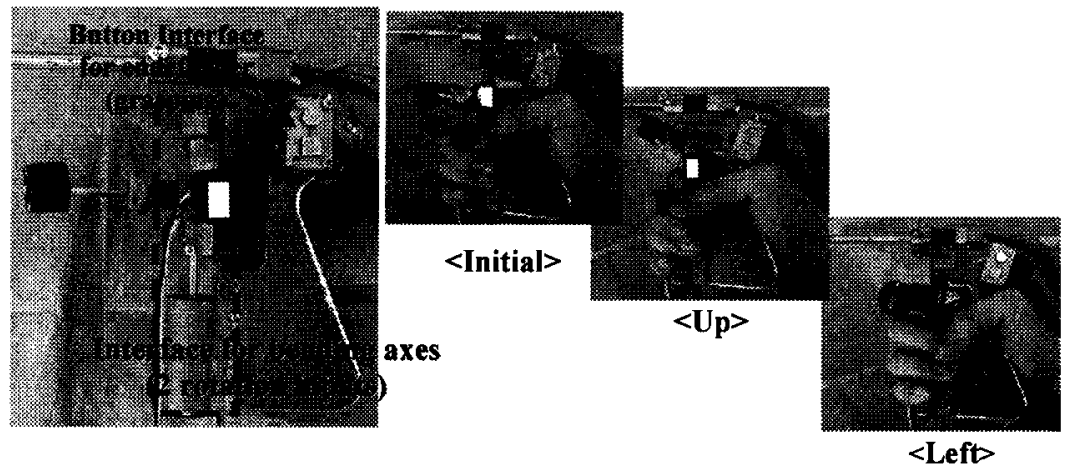

Fig. 8. The Prototype of New Interface for Multi-DOF Forceps Manipulator

The mechanism of the interface is explained in Fig. 9. The characteristics of the manipulator are presented in Table 1.

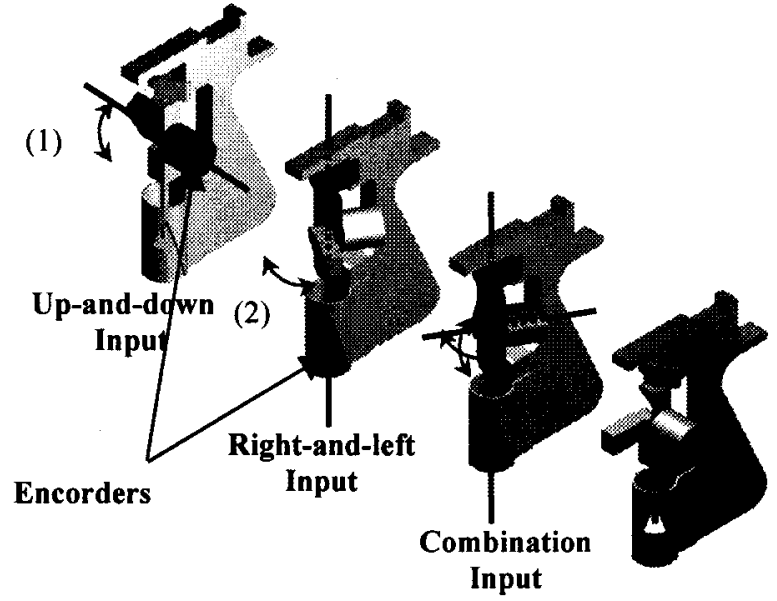

Fig. 9. Input Mechanism of the New Interface

Table 1. Specification of the bending forceps manipulator system

\begin{tabular}{cc}
\hline Range of actions (DOF) & 2 bending and 1 grasping \\
Moving range & $0-60^{\circ}$ (bending) $/ 0-60^{\circ}$ (grasping) \\
Diameter & $5 \mathrm{~mm}$ \\
Size & $345 \mathrm{~mm}$ (l) $\times 135 \mathrm{~mm}(\mathrm{~h}) \times 15 \mathrm{~mm}(\mathrm{~d})$ \\
Weight & $250 \mathrm{~g}$ (Multi-DOF forceps) \\
Material & Stainless (SUS304) \\
\hline
\end{tabular}




\section{Experimental Results}

\subsection{Mechanical Characteristics}

Firstly, we checked the characteristics of bending movements. The movement characteristics have hysteresis, with large backlash due to the stretching and friction of the wires. The difference between $\phi 6 \mathrm{~mm}$ drive and $\phi 5 \mathrm{~mm}$ drive is mainly caused by the efficiency fall of wire driving force and deterioration of a wire due to the mechanism miniaturized. The Teflon tube removal and the errors in manufacture and assembly are the biggest effect to the bending drive. Due to these problems, the characteristics of the bending drive $(\phi 5 \mathrm{~mm})$ go little worse than that of $\phi 6 \mathrm{~mm}$. But these problems will be solved through the reshaping the wire drive system and manufacture method and by using more appropriate materials.

\subsection{Testing in a Semi-clinical Situation}

We tested the clinical performance of this Multi-DOF forceps manipulator by using it in a semi-clinical situation. The surgeon carried out trial operations (grasping, bending, extension, and ligation, etc.). This test is carried out on the training tool set for laparoscopic surgery. (Fig. 12)

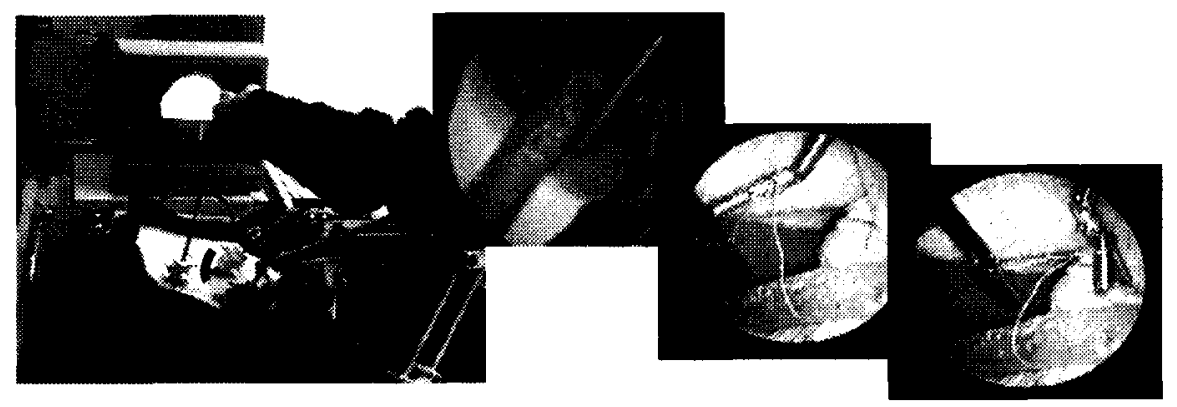

Fig. 12. Testing overviews of the Multi-DOF forceps manipulator system

As a result of having used Multi-DOF forceps, the advantages was recognized compared with a general forceps which was a straight, in the following points;

(1) Turning thread around the total bile duct and detaining thread:

The surgeon can send thread easily without excessive force because the "circumference" operation can be carried out.

(2) Winding thread around the forceps and catching another end of the thread and pulling it (ligation):

The surgeon can accomplish the ligation easier when the range between two forceps is nearly plumb. Multi-DOF forceps can control the range more easily than the commercial straight forceps. Using straight one, the ability of controlling the range depends on the positions of trochars.

In addition, it is easy to start an operation order and is hard to make a mistake in using 2 axes stick type interface compared with a button type, and the possibility of improvement of the surgeon's dexterity was recognized. 
However, the poor responses of movement (due to the backlash of the wire drive system) become more serious than the case of $6 \mathrm{~mm}$ forceps manipulator. We must solve this problem for the establishment of the total forceps manipulator system with additional DOF and which provide surgeons high performance of laparo-procedure (with high dexterity, high accuracy, etc).

In this experiment, although there were indeed problems, the basic functions of this Multi-DOF forceps manipulator with new mechanism were confirmed as sufficiently useful for laparoscopic surgery.

\section{Discussion}

\subsection{Multi-DOF Forceps Manipulator with New Mechanisms}

The new mechanism of $\phi 5 \mathrm{~mm}$ forceps manipulator has some problems in drive characteristics. Through the establishment of the high precision manufacturing and assembly of the small components and the adoption of the materials and design appropriate for the mechanism, we will develop the small Multi-DOF Forceps Manipulator System with high performances. The performance of new Interface is confirmed as useful basically in the evaluation. The improvement points considered to be necessary for this system are;

- Heaviness \& lash of a 2 axis stick interface

When an axis of interface is too light and too sensitive, maintenance of bending state becomes difficult. In opposite, when too heavy and too insensitive, the operation with high accuracy and high response cannot be carried out.

- Optimization of the grip shape

It is necessary to drive the bending axes and the other DOF of forceps simultaneously. Therefore, the steady holding of forceps grip with three finger is very important. We must consider the optimization of the grip shape or the development of new holder that support the steady holding of this manipulator.

- The Interface for grasping

In this paper, we only developed the new interface for bending axes. If pursuing the intuitional drive, it is desirable to establish the interface for grasping that imitated movement of "grasping" instead of using two buttons.

- More convictive evaluation

In this experiment, we tested the system in very limited case and method. It's of course not enough to discriminate the effectiveness of this system in real surgery. We need some more quantitative evaluations and comparison of the system to conventional laparoscopic instruments and many existing active forceps research.

\subsection{Future Work from Additional DOF to Additional Function}

This Multi-DOF Forceps Manipulator System is developed under the desire for additional DOF of surgical tools in MIS. And there is another strong desire for new surgical tools. That is the surgical tool with "Additional Function". 
We are now planning and prototyping a Multi-DOF Bipolar Coagulator Manipulator. Using this manipulator, the surgeons can possess the wide surgical field, wide surgical approach pass, and wide surgical procedure, and they can be free from many limitations in laparoscopic surgery, e.g. due to the necessity of retaining the surgical fields, changing many forceps according as situations, and the difficulty in management of vessels and vascular (clipping or ligation).

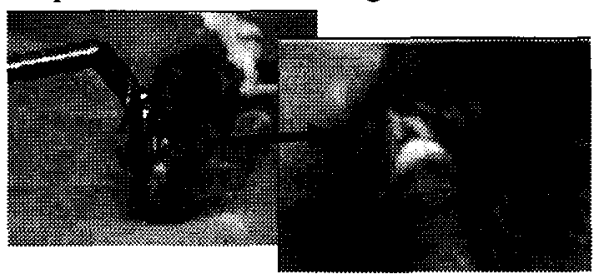

Fig. 13. Multi-DOF Bipolar Coagulator Manipulator System

\section{Conclusion}

We have developed a Multi-DOF forceps manipulator with new two mechanisms. Through the testing in a semi-clinical situation, although some problems were found, the effectiveness of this manipulator was clearly demonstrated. Especially, as for the stick type interface, the effectiveness of improvement of operability was recognized enough. With the improvement of the DOF arrangement and the stability of bending drive, the construction of the new interface for surgical tools will be possible.

\section{Acknowledgements}

This study was partly supported by the Research for the Future Program JSPS-RFTF 96P00801 \& JSPS-RFTF 99I00904, and by Grant-in-Aid for Scientific Research (JSPS Fellow).

\section{References}

1. Rininsland H.: ARTEMIS. A telemanipulator for cardiac surgery, European Journal of Cardio-Thoracic Surgery 16(2) (1999) 106-111

2. Shennib H., Bastawisy A., McLoughlin J., Moll F.: Robotic computer-assisted telemanipulation enhances coronary artery bypass, Journal of Thoracic \& Cardiovascular Surgery 117(2) Feb (1999) 310-313

3. Cohn, M., L. S. Crawford, J. M. Wendlandt, S. S. Sastry: Surgical Applications of MilliRobots, Journal of Robotic Systems 12(6) (1995) 401-416

4. Steve Charles, Hari Das, Tim Ohm, Curtis Boswell, Guillermo Rodriguez, Robert Steele, Dan Istrate: Dexterity-enhanced Telerobotic Microsurgery Proceedings of 8th International Conference on Advanced Robotics (ICAR '97) (1997) 5-10

5. Ikuta K., Kato T., Nagata S.: Micro active forceps with optical fiber scope for intra-ocular microsurgery, Proceedings of the IEEE Micro Electro Mechanical Systems (MEMS) (1996) 456-461

6. Nakamura R. et al: Multi-DOF Forceps Manipulator System for Laparoscopic Surgery, Proceeding of Medical Image Computing and Computer-Assisted Intervention - MICCAI 2000 ,Springer, LNCS, Vol.1935, (2000) 653-660

7. Hashimoto D.: Gasless Laparoscopic Surgery World Scientific Publishing (1995)

8. Breedveld P., Stassen H., Meijer D., Jakimowicz J.: Manipulation in Laparoscopic surgery: Overview of Impeding Effects and Supporting Aids, Joumal of Laparoendoscopic \& Advanced Surgical Techniques 9(6) (1999) 469-480 